\title{
Efecto de la dieta cetogénica baja en calorías sobre la composición corporal en adultos con sobrepeso y obesidad: revisión sistemática y metaanálisis
}

\author{
Effect of a low-calorie ketogenic diet on body composition in overweight and \\ obese adults: $A$ systematic review and meta-analysis \\ Efeito de uma dieta cetogênica de baixa caloria na composição corporal em adultos com \\ sobrepeso e obesos: uma revisão sistemática e meta-análise
}

Gustavo Alfonso Díaz-Muñoz ${ }^{* 1}$, Ángela María Castañeda-Gómez², María Paula Belalcázar-Monsalve²,
Juan Pablo Zambrano-Salazar², María Camila Bautista-Velandia'², Felipe Ballesteros-Arbeláez².

Recibido: 17 de enero de 2021. Aceptado para publicación: 2 de marzo de 2021.

Publicado en línea: 5 de marzo de 2021.

https://doi.org/10.35454/rncm.v4n3.273

\begin{abstract}
Resumen
Introducción: la dieta cetogénica baja en calorías (DC) se conoce por su efecto para reducir el peso corporal. La pérdida de peso saludable debe reducir la grasa corporal y mantener la masa muscular, pero los metaanálisis previos no han evaluado estos cambios. El objetivo de este metaanálisis fue cuantificar el efecto de la dieta cetogénica comparada con una dieta hipocalórica $(\mathrm{DH})$, sobre los cambios de composición corporal en adultos con exceso de peso.
\end{abstract}

Métodos: se realizó una revisión sistemática y metaanálisis según la metodología Cochrane. Se consultaron 5 bases de datos usando los términos "Body Weight" OR "Body Composition" AND "Diet, Ketogenic". Se incluyeron estudios experimentales controlados, seguimiento $\geq 4$ semanas realizados en adultos con IMC $\geq 25 \mathrm{~kg} / \mathrm{m}^{2}$ y desenlaces de peso y composición corporal.

Resultados: la búsqueda arrojó 492 artículos, de los cuales se seleccionaron 10 para el metaanálisis y solo 3 presentaron bajo riesgo de sesgo. El metaanálisis indicó que la dieta cetogénica, respecto a la dieta hipocalórica, promueve mayor pérdida de peso $(-6,9 \mathrm{~kg}$, intervalo de confianza [IC] $95 \%:-10,4 ;-3,3)$, de índice

\section{Summary}

Introduction: low-calorie ketogenic diet is known for its effect on reducing body weight. Healthy weight loss should reduce the body fat and maintain the muscle mass, but previous meta-analyses have not evaluated these changes. The objective of this meta-analysis was to quantify the effect of ketogenic diet compared to a hypocaloric diet, on changes in body composition in overweight adults.

Methods: A systematic review and meta-analysis was carried out according to Cochrane methodology. Five databases were consulted using the terms "Body Weight" OR "Body Composition" AND “Diet, Ketogenic". Controlled experimental studies, with a follow-up $\geq 4$ weeks conducted in adults with body mass index (BMI) $\geq 25 \mathrm{~kg} / \mathrm{m}^{2}$ and reported weight and body composition outcomes were included.

Results: The search yielded 492 articles, of which 10 were selected for meta-analysis and 3 had low risk of bias. Our meta-analysis indicates that ketogenic diet promotes greater weight loss than hypocaloric diet (-6,9 kg; 95\% confidence interval [CI]: -10.4; $-3.3)$, BMI (-1.6 kg/m²; 95\% Cl: $-3.0 ;-0.2)$, and fat mass $(-0.8 \mathrm{~kg} ; 95 \% \mathrm{Cl}:-1.1 ;-0.5)$. Not enough information was found to inquire about muscle mass and exercise. The risks

\section{Resumo}

Introdução: a dieta cetogênica baixa em calorías é conhecida por seu efeito na redução no peso corporal. A perda de peso saudável deve reduzir a gordura corporal e manter a massa muscular, porém em meta-análises anteriores essas variáveis não foram avaliadas. $\mathrm{O}$ objetivo desta meta-análise foi avaliar o efeito da dieta cetogênica em comparação com uma dieta hipocalórica, nas mudanças da composição corporal em adultos com excesso de peso.

Métodos: foi realizada uma revisão sistemática e meta-análise de acordo com a metodologia Cochrane. Cinco bases de dados foram consultadas, utilizando os termos ("Body Weight" OR "Body Composition") AND ("Diet, Ketogenic"). Foram incluídos estudos experimentais controlados, com acompanhamento de quatro semanas ou mais, realizados em adultos com IMC $\geq 25 \mathrm{~kg} / \mathrm{m}^{2}$, valores de peso e composição corporal.

Resultados: Foram encontrados 492 artigos, dos quais 10 foram selecionados para meta-análise e apenas três tinham baixo risco de viés. A meta-análise indicou que a dieta cetogênica em comparação com dieta hipocalórica promoveu maior perda de peso $(-6,9 \mathrm{~kg} 95 \% \mathrm{Cl}-10,4 ;-3,3)$, 
de masa corporal (IMC; $-1,6 \mathrm{~kg} / \mathrm{m}^{2}$; IC 95 $\%:-3,0 ;-0,2)$ y masa grasa $(-0,8 \mathrm{~kg} ;$ IC 95 $\%:-1,1 ;-0,5)$. No se encontró suficiente información para indagar sobre la masa muscular. Los riesgos de sesgo junto con el sesgo de publicación y de reporte podrían estar sobreestimando el efecto de la dieta cetogénica.

Conclusiones: en adultos con exceso de peso y con seguimiento hasta de 12 meses, la dieta cetogénica, comparada con la dieta hipocalórica, presentó mayor pérdida de peso, disminución del IMC y reducción en la masa grasa, pero podría estar sobreestimado por sesgos de publicación o reporte. Por lo anterior, se considera innecesario emplear la dieta cetogénica para el control del peso corporal.

Palabras clave: dieta cetogénica, sobrepeso, obesidad, metaanálisis. of bias together with publication and reporting bias could be overestimating the effect of ketogenic diet.

Conclusions: In overweight adults with a follow-up of up to 12 months, ketogenic diet compared with HD showed loss of weight, BMI and fat mass, but it could be overestimated due to publication and reporting biases. Therefore, it is considered unnecessary to use ketogenic diet to control body weight.

Keywords: Diet; Ketogenic; Overweight; Obesity; Meta-Analysis. maior diminuição do IMC $\left(-1,6 \mathrm{~kg} / \mathrm{m}^{2}\right.$ IC $95 \%-3,0 ;-0,2)$, e da gordura corporal (- 0,8 kg IC $95 \%-1,1 ;-0,5)$. Não foram encontrados dados suficientes em relação a massa muscular. Os riscos de viés, juntamente com o viés de publicação e viés de reporte de resultado poderiam estar superestimando o efeito da dieta cetogênica.

Conclusões: Em adultos com excesso de peso e com acompanhamento de até 12 meses, a dieta cetogênica em comparação com a dieta hipocalórica mostrou perda de peso, de IMC e de gordura corporal, pudendo estar superestimada devido a vieses de publicação e vieses de reporte de resultado. Portanto, considera-se desnecessário o uso da dieta cetogênica para o controle do peso corporal.

Palavras-chave: dieta cetogênica; sobrepeso; obesidade; meta-análise.
Instituto de investigación de nutrición, genética y metabolismo. Facultad de Medicina, Universidad El Bosque, Bogotá, Colombia.
2 Semillero de investigación de nutrición, genética y cáncer. Facultad de Medicina, Universidad El Bosque, Bogotá, Colombia.

\section{INTRODUCCIÓN}

La obesidad representa un problema de salud pública a nivel mundial debido a su asociación con enfermedades crónicas no transmisibles y reducción de la calidad y expectativa de vida ${ }^{(1,2)}$. La estrategia terapéutica reconocida es el tratamiento médico-nutricional, que incluye dietoterapia, fármacos y la cirugía bariátrica ${ }^{(3-7)}$. El tratamiento médico-nutricional, bajo un enfoque multidisciplinario, busca lograr un estilo de vida saludable en el paciente con obesidad. A través de cambios en la alimentación y la actividad física se busca la pérdida de peso, principalmente grasa corporal, al tiempo que se mantiene la masa muscular. Esto debe considerarse en cualquier paciente que presente pérdida de peso, debido a que la masa muscular tiene funciones de locomoción, postura, fuerza, reserva de aminoácidos y metabolismo; si hay una pérdida de masa muscular, se expone al individuo a mayor riesgo de mortalidad, fragilidad, resistencia a la insulina, disfunciones metabólicas, caídas, lesiones articulares y sarcopenia ${ }^{(8-10)}$.
Algunas dietas, como la dieta cetogénica baja en calorías, han surgido como estrategias en el manejo de la obesidad para lograr pérdidas sostenidas y el mantenimiento de su peso corporal ${ }^{(11)}$. La dieta cetogénica también se utiliza en pacientes que se preparan para cirugía bariátrica ${ }^{(6)}$ y ha presentado beneficios sobre el perfil lipídico, glucemia y composición corporal ${ }^{(12,13)}$.

La dieta cetogénica se caracteriza por un protocolo de 3 fases. La primera fase, fase activa, consiste en una ingesta de comidas con elevada cantidad de proteína (usualmente un aislado de proteína animal o vegetal) y vegetales con bajo índice glucémico 5 veces al día; en esta fase, los pacientes empiezan con una restricción tanto calórica como de carbohidratos, con una cantidad de 0,8 a $1,2 \mathrm{~g} / \mathrm{kg} /$ día de proteína y una duración media de 12 semanas, la cual se asocia con el incremento de producción de cuerpos cetónicos. La segunda fase es la de estabilización metabólica, en la que se sustituye el aislado de proteína en el almuerzo o cena por una proteína natural como carne o pescado. La última fase corresponde a la de mantenimiento, en la que hay sus- 
titución de la segunda porción de proteína aislada por una natural baja en grasa ${ }^{(11,14)}$.

Los mecanismos por los cuales la dieta cetogénica contribuye a la pérdida de peso son un mayor uso de la grasa en ausencia o déficit de carbohidratos, el efecto saciante y el índice glucémico. Ante el déficit de carbohidratos, se desencadenan mecanismos contrarreguladores que promueven la lipólisis y producción de cuerpos cetónicos. El efecto saciante se debe a que las grasas y las proteínas permanecen en el estómago durante más tiempo, liberación de colecistoquinina (hormona supresora del apetito), liberación de $\beta$-hidroxibutirato (capacidad para inhibir directamente el centro del apetito), sensibilidad aumentada del sistema nervioso central (SNC) hacia la leptina y descenso en las concentraciones plasmáticas posprandiales de grelina. Finalmente, el bajo índice glucémico de la dieta cetogénica disminuye la fluctuación en las concentraciones plasmáticas de glucosa, de manera que se reduce el apetito al evitarse episodios de hipoglucemia ${ }^{(15,16)}$.

A pesar de lo anterior, no hay evidencia acerca de los efectos de la dieta cetogénica sobre la composición corporal cuando se compara con dietas balanceadas hipocalóricas (dieta hipocalórica) ${ }^{(17-19)}$. Los metaanálisis previos han identificado que, a largo plazo $(>12$ meses), la dieta cetogénica es poco efectiva para la pérdida de peso $^{(2,20)}$, la cual se explica principalmente por la pérdida de masa grasa ${ }^{(21)}$. La revisión de Di Rosa y colaboradores ${ }^{(16)}$ es la única que ha evaluado otros componentes de la composición corporal, y se indicó que hay pérdida de masa grasa y conservación de la masa magra. Sin embargo, esta revisión incluyó estudios recientes (2014-2019), los cuales discrepan entre sí y no hay un valor agrupado que permita sustentar las afirmaciones.

Por tanto, el objetivo de esta revisión fue cuantificar el efecto de la dieta cetogénica comparada con la dieta hipocalórica, sobre los cambios de peso y composición corporal en adultos con exceso de peso. Realizar esta revisión sistemática y metaanálisis incrementaría la buena práctica basada en la evidencia y recomendaría el rumbo de las próximas investigaciones.

\section{MÉTODOS}

El diseño de estudio es una revisión sistemática y metaanálisis en el que se empleó la metodología de revisiones sistemáticas de Cochrane y se reportó la información según las recomendaciones PRISMA.

\section{Estrategia de búsqueda}

Se realizó una revisión de la literatura en 5 buscadores: PubMed, EMBASE, BVS, Scielo y Google Scholar. La búsqueda de los artículos fue realizada con un intervalo de tiempo del 1 de enero de 1990 hasta el 26 febrero de 2020 y se restringió a los idiomas español e inglés. Se emplearon términos estandarizados en cada base de datos, aplicando el algoritmo de búsqueda: ((("Body Weight") OR "Body Composition")) AND "Diet, Ketogenic") AND “Adult”.

\section{Selección de los estudios}

Los criterios de inclusión para los artículos fueron: estudios experimentales con grupo control (sin intervención o dieta baja en calorías), en humanos, en paralelo, con o sin aleatorización y con mínimo 4 semanas de seguimiento. En caso de estudios con más de una publicación, se tomó el de mayor tiempo de seguimiento. En cuanto a la población, se tuvo en cuenta estudios realizados en adultos y con un IMC $>25 \mathrm{~kg} / \mathrm{m}^{2}$. La intervención fue la dieta cetogénica baja en calorías ( $<20 \%$ o $<50$ g carbohidratos/día) $\mathrm{y}$ los desenlaces de interés fueron el cambio de peso corporal, IMC y composición corporal medida por absorsiometría de rayos X de energía dual (DXA, por sus siglas en inglés) o impedancia bioeléctrica (BIA, por sus siglas en inglés).

Por otro lado, se excluyeron estudios relacionados con enfermedades autoinmunes (esclerosis), enfermedades neurológicas (epilepsia, migraña, Alzheimer, demencia, esquizofrenia, etc.), cirugías metabólicas previas, alto rendimiento físico, atletas, militares, cáncer, tratamiento de hígado graso no alcohólico, enfermedad renal, lactancia, suministro de la dieta por vía enteral o parenteral y tratamiento de pérdida de peso con medicamentos.

Para la selección de artículos se aplicaron los criterios de selección en el título y resumen de manera individual e independiente por cinco investigadores. Luego se realizó un consenso para determinar los estudios preseleccionados. Los desacuerdos fueron resueltos con el sexto investigador. De los artículos preseleccionados se revisó el artículo completo para aplicar los criterios de selección de manera individual e independiente por cinco investigadores, posteriormente los desacuerdos se resolvieron por consenso. 


\section{Extracción de información}

Toda la información se extrajo de los artículos publicados, de los protocolos disponibles y, en algunos casos, por contacto directo vía e-mail con un autor del artículo. Si la investigación contaba con más de dos grupos experimentales, se seleccionaron los más relacionados con el objetivo de la revisión.

En una hoja de Excel se organizaron los artículos que los autores consideraron útiles en este estudio. Se extrajo la siguiente información: año de publicación, país, sexo, edad, descripción de la dieta cetogénica, descripción de la dieta hipocalórica, tamaño de muestra, tiempo de seguimiento máximo y mediciones de composición corporal (peso, IMC, masa grasa, masa libre de grasa y masa muscular).

Respecto a la evaluación del riesgo de sesgo, se empleó el instrumento RoB2 de la Colaboración Cochrane para el desenlace de peso, IMC y composición corporal, debido a que son variables interrelacionadas. La evaluación se hizo entre tres investigadores de manera individual e independiente, considerando en la evaluación que la naturaleza de las intervenciones no permite un enmascaramiento al paciente y a los investigadores.

\section{Análisis estadístico}

Los datos de los desenlaces fueron de tipo continuo y se reportaron como el valor al final del seguimiento, $o$ como diferencia entre el valor final y el valor inicial. Las medidas del efecto fueron diferencia de medias (para el peso corporal e IMC) y diferencia de medias estandarizadas (para masa grasa, masa libre de grasa y masa muscular). El peso de los estudios se determinó mediante el método de inverso de la varianza.

La heterogeneidad estadística se midió mediante una prueba $\mathrm{Chi}^{2}$, asumiendo como heterogeneidad valores de $p<0,1$; el porcentaje de variabilidad se midió con el estadístico $\mathrm{I}^{2}$, asumiendo un $\mathrm{I}^{2}>50 \%$ como heterogeneidad significativa. Se empleó un metaanálisis de efectos aleatorios para incorporar la heterogeneidad de los estudios en el efecto combinado. Además, se realizaron análisis por subgrupos según restricción calórica (mayor en la dieta hipocalórica y mayor en la dieta cetogénica), duración de la intervención ( $<6$ meses y $\geq 6$ meses) y combinación de la intervención con ejercicio (solo dieta o dieta más ejercicio). La evaluación del sesgo del informe se realizó mediante el gráfico de embudo y la prueba de Egger. Todos los análisis y gráficos se realizaron en RevMan 5.4 (Colaboración Cochrane).

\section{RESULTADOS}

\section{Estudios incluidos}

La búsqueda arrojó un total de 483 artículos; de estos, se preseleccionaron 42 artículos según el título y resumen, y a partir de la lectura completa del artículo, se seleccionaron 10 para los análisis cuantitativos ${ }^{(18,22-30)}$ (Figura 1).

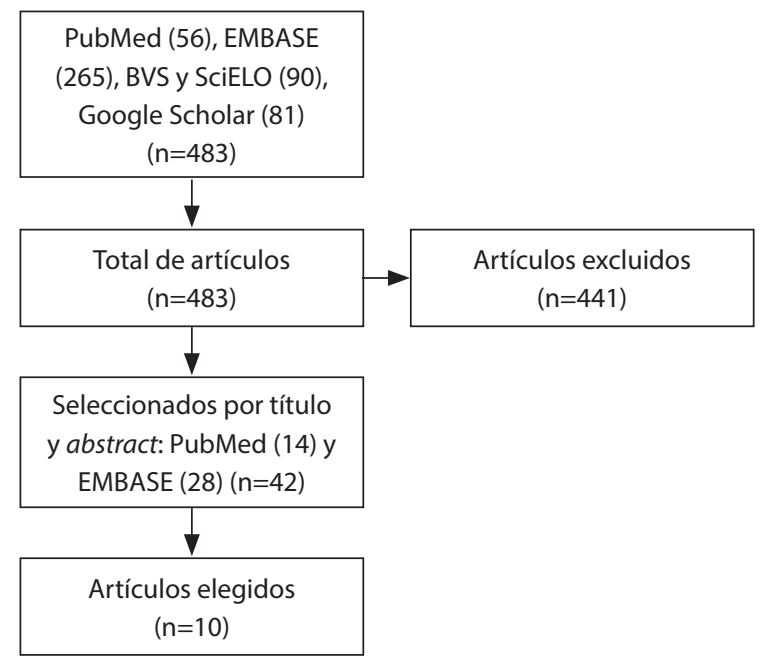

Figura 1. Diagrama de flujo de la búsqueda y selección de artículos.

En total, 369 participantes recibieron dieta cetogénica baja en calorías y 344 recibieron dieta hipocalórica balanceada. Un estudio fue no aleatorizado, la edad promedio fue superior a 35 años y únicamente dos artículos no reportaron cointervenciones; tanto la implementación de la dieta cetogénica como de la dieta hipocalórica varió entre las investigaciones (Tabla 1). En cuanto a la evaluación del riesgo de sesgo, tres publicaciones presentaron bajo riesgo de sesgo y los sesgos más predominantes fueron la adherencia a la intervención, la medición del desenlace y el reporte selectivo (Tabla 2).

\section{Cambios de peso corporal}

El peso corporal final se reportó en 9 publicaciones y 6 de estas describen una mayor pérdida de peso en la dieta cetogénica que en la hipocalórica. En el metaanálisis global se encontró que en la dieta cetogénica se pueden perder 6,9 kg más (IC del $95 \% 10,4$ a 3,3) respecto a la dieta hipocalórica, lo cual no cambia con la realización de ejercicio físico (Figura 2). Además, la dieta cetogénica impone una mayor pérdida de peso 
Tabla 1. Descripción de los estudios incluidos

\begin{tabular}{|c|c|c|c|c|c|c|c|}
\hline Estudio & $\begin{array}{l}\text { Tipo de } \\
\text { experimento } \\
\text { y duración } \\
\text { en meses }\end{array}$ & $\begin{array}{l}\text { Población } \\
\text { cetogénica }\end{array}$ & $\begin{array}{l}\text { Población } \\
\text { hipocalórica }\end{array}$ & $\begin{array}{l}\text { Método } \\
\text { valoración } \\
\text { composición } \\
\text { corporal }\end{array}$ & Dieta cetogénica & Dieta hipocalórica & Co intervenciones \\
\hline $\begin{array}{l}\text { Perticone y } \\
\text { colaboradores } \\
(2019)^{(22)} \\
\text { Italia }\end{array}$ & $\begin{array}{l}\text { Controlado } \\
\text { aleatorizado } \\
12\end{array}$ & $\begin{array}{l}\text { H } 14 \\
\text { M } 14 \\
42.6 \pm 6,6 \text { años } \\
\text { IMC } 40,5 \pm 10,8 \\
\mathrm{~kg} / \mathrm{m}^{2}\end{array}$ & $\begin{array}{l}\text { H } 18 \\
\text { M } 10 \\
50,9 \pm 13,3 \text { años } \\
\text { IMC } 38,8 \pm 4,5 \\
\mathrm{~kg} / \mathrm{m}^{2}\end{array}$ & BIA & $\begin{array}{l}\text { Inicio: } 600-800 \mathrm{kcal} / \mathrm{dí} \\
\text { Baja en calorías: } 1000-1500 \mathrm{kcal} / \mathrm{día} \\
\text { Mantenimiento: } 1500-2000 \mathrm{kcal} / \mathrm{dí} a \\
50 \%-60 \% \text { proteína } \\
20 \%-30 \% \text { lípidos } \\
20 \% \text { carbohidratos }\end{array}$ & $\begin{array}{l}\text { Dieta Mediterránea } \\
\text {-500 kcal/día } \\
10 \%-15 \% \text { proteína } \\
25 \%-30 \% \text { lípidos } \\
55 \%-60 \% \text { carbohidratos }\end{array}$ & No reporta \\
\hline $\begin{array}{l}\text { Sajoux y } \\
\text { colaboradores } \\
(2019)^{(23)} \\
\text { España }\end{array}$ & $\begin{array}{l}\text { Controlado } \\
\text { aleatorizado } \\
6\end{array}$ & $\begin{array}{l}\text { H } 8 \\
\text { M } 12 \\
47,2 \pm 10,2 \text { años } \\
\text { IMC } 35,5 \pm 4,4 \\
\mathrm{~kg} / \mathrm{m}^{2}\end{array}$ & $\begin{array}{l}\text { H } 10 \\
\text { M } 10 \\
49,9 \pm 9,3 \text { años } \\
\text { IMC } 34,6 \pm 3,5 \\
\mathrm{~kg} / \mathrm{m}^{2}\end{array}$ & DXA & $\begin{array}{l}\text { Método PnK } \\
\text { Inicio: } 600-800 \mathrm{kcal} / \text { día } \\
\text { Baja en calorías: } 800-1500 \mathrm{kcal} / \text { día } \\
\text { Mantenimiento: } 1500-2000 \mathrm{kcal} / \text { día }\end{array}$ & $\begin{array}{l}\text { Inicio ( } 2 \text { meses): reducción } 30 \% \\
-600 \text { a }-800 \text { kcal/día } \\
40 \%-55 \% \text { carbohidratos } \\
30 \% \text { lípidos } \\
15 \%-30 \% \text { proteína }\end{array}$ & No reporta \\
\hline $\begin{array}{l}\text { Saslow y } \\
\text { colaboradores } \\
(2017)^{(24)} \\
\text { Estados } \\
\text { Unidos } \\
\end{array}$ & $\begin{array}{l}\text { Controlado } \\
\text { aleatorizado } \\
12\end{array}$ & $\begin{array}{l}\text { H } 7 \\
\text { M } 9 \\
64,8 \pm 7,7 \text { años } \\
\text { IMC } 36,4 \pm 7,9 \\
\mathrm{~kg} / \mathrm{m}^{2}\end{array}$ & $\begin{array}{l}\mathrm{H} 2 \\
\mathrm{M} 16 \\
55,1 \pm 13,5 \text { años } \\
\mathrm{IMC} 37 \pm 6,4 \\
\mathrm{~kg} / \mathrm{m}^{2}\end{array}$ & Antropometría & $\begin{array}{l}-500 \mathrm{kcal} / \mathrm{día} \\
20-50 \mathrm{~g} \text { carbohidratos }\end{array}$ & $\begin{array}{l}\text { Guías ADA } \\
-500 \mathrm{kcal} / \text { día } \\
45 \%-50 \% \text { carbohidratos }\end{array}$ & $\begin{array}{l}19 \text { clases de mindfulness, } \\
\text { aumentar horas de sueño y } \\
\text { ejercicio }\end{array}$ \\
\hline $\begin{array}{l}\text { Moreno y } \\
\text { colaboradores } \\
(2016)^{(18)} \\
\text { España }\end{array}$ & $\begin{array}{l}\text { Controlado } \\
\text { aleatorizado } \\
12\end{array}$ & $\begin{array}{l}\text { H } 5 \\
\text { M } 22 \\
44,4 \pm 8,6 \text { años } \\
\text { IMC } 35,1 \pm 4,5 \\
\mathrm{~kg} / \mathrm{m}^{2}\end{array}$ & $\begin{array}{l}\mathrm{H} 1 \\
\mathrm{M} 25 \\
46,3 \pm 9,3 \text { años } \\
\mathrm{IMC} 35.1 \pm 5.3 \\
\mathrm{~kg} / \mathrm{m}^{2}\end{array}$ & DXA & $\begin{array}{l}\text { Método Pronokal } \\
\text { Activa: } 600-800 \mathrm{kcal} / \text { día } \\
25 \% \text { carbohidratos, } 11 \% \text { lípidos; } \\
0,8-1,2 \mathrm{~g} / \mathrm{kg} / \text { día proteína } \\
\text { Reeducación: } 800-1500 \mathrm{kcal} / \mathrm{día} \\
\text { Mantenimiento: } 1500-2250 \mathrm{kcal} / \text { día }\end{array}$ & $\begin{array}{l}\text {-10\% GCT; } 1400-1800 \text { kcal/ } \\
\text { día } \\
45 \%-55 \% \text { carbohidratos } \\
15 \%-25 \% \text { proteínas } \\
25 \%-35 \% \text { lípidos }\end{array}$ & $\begin{array}{l}12 \text { reuniones grupales, } \\
\text { sesiones de consejería } \\
\text { individuales e incentivo para } \\
\text { ejercitarse de manera regular } \\
\text { de acuerdo con un programa } \\
\text { establecido de ejercicio }\end{array}$ \\
\hline $\begin{array}{l}\text { Goday y } \\
\text { colaboradores } \\
(2016)^{(25)} \\
\text { España }\end{array}$ & $\begin{array}{l}\text { Controlado } \\
\text { aleatorizado } \\
4\end{array}$ & $\begin{array}{l}\text { H } 15 \\
\text { M } 30 \\
54,9 \pm 8,8 \text { años } \\
\text { IMC } 33,3 \pm 1,5 \\
\mathrm{~kg} / \mathrm{m}^{2}\end{array}$ & $\begin{array}{l}\text { H } 16 \\
\text { M } 28 \\
54,2 \pm 8 \text { años } \\
\text { IMC } 32,9 \pm 1,6 \\
\mathrm{~kg} / \mathrm{m}^{2}\end{array}$ & Antropometría & $\begin{array}{l}\text { Método Diaprokal } \\
\text { Fase activa: } 600-800 \mathrm{kcal} / \text { día } \\
<50 \mathrm{~g} \text { carbohidratos; } 10 \mathrm{~g} \text { aceite } \\
\text { oliva; 0,8-1,2 g/kg/día proteína } \\
\text { Fase metabólica: } 800-1500 \mathrm{kcal} / \text { día } \\
\text { Fase mantenimiento: } 1500-2250 \\
\text { kcal/día }\end{array}$ & $\begin{array}{l}\text { Guías ADA } \\
\text {-500-1000 kcal/día } \\
<30 \% \text { lípidos } \\
10 \%-20 \% \text { proteína } \\
45 \%-60 \% \text { carbohidratos }\end{array}$ & $\begin{array}{l}\text { Reuniones grupales y } \\
\text { recomendaciones. } \\
9 \text { sesiones individuales. } \\
\text { Consejería individual. } \\
\text { Apoyar las modificaciones } \\
\text { en el estilo de vida y } \\
\text { comportamiento }\end{array}$ \\
\hline $\begin{array}{l}\text { Rubini y } \\
\text { colaboradores } \\
(2015)^{(26)} \\
\text { Italia }\end{array}$ & $\begin{array}{l}\text { Controlado } \\
\text { aleatorizado } \\
2\end{array}$ & $\begin{array}{l}\text { M } 16 \\
51,4 \pm 12,4 \text { años } \\
\text { IMC } 29,3 \pm 2,8 \\
\mathrm{~kg} / \mathrm{m}^{2}\end{array}$ & $\begin{array}{l}\text { M } 16 \\
44,7 \pm 13,9 \text { años } \\
\text { IMC } 27,5 \pm 2,8 \\
\mathrm{~kg} / \mathrm{m}^{2}\end{array}$ & BIA & $\begin{array}{l}\text { Fase I: } 20 \text { días: } 848 \text { kcal/día } \\
43 \% \text { proteína } \\
14 \% \text { carbohidratos y } 43 \% \text { lípidos. } \\
\text { Fase Il: } 20 \text { días. } 938 \mathrm{kcal} / \text { día. } 28 \% \\
\text { proteína, } 34 \% \text { carbohidratos y } 38 \% \\
\text { lípidos. } \\
\text { Fase Ill: } 20 \text { días. } 1400 \mathrm{kcal} / \text { día. } 15 \% \\
\text { proteína, } 60 \% \text { carbohidratos y } 35 \% \\
\text { lípidos. }\end{array}$ & $\begin{array}{l}\text { Dieta mediterránea } \\
\text { Fase I: } 20 \text { días. } 1200 \mathrm{kcal} / \\
\text { día. } 15 \% \text { proteína, } 60 \% \\
\text { carbohidratos y } 25 \% \text {. } \\
\text { Fase II: } 20 \text { días. } 1400 \mathrm{kcal} / \\
\text { día. } 15 \% \text { proteína, } 60 \% \\
\text { carbohidratos y } 25 \% \text { lípidos. } \\
\text { Fase III: } 20 \text { días. } 1400 \mathrm{kcal} / \\
\text { día. } 15 \% \text { proteína, } 60 \% \\
\text { carbohidratos y } 35 \% \text { lípidos }\end{array}$ & $\begin{array}{l}\text { Sugerencia de actividad } \\
\text { física moderada y uso de } \\
\text { infusiones a lo largo del día }\end{array}$ \\
\hline
\end{tabular}


Tabla 1. Descripción de los estudios incluidos (continuación)

\begin{tabular}{|c|c|c|c|c|c|c|c|}
\hline Estudio & $\begin{array}{l}\text { Tipo de } \\
\text { experimento } \\
\text { y duración } \\
\text { en meses }\end{array}$ & $\begin{array}{l}\text { Población } \\
\text { cetogénica }\end{array}$ & $\begin{array}{l}\text { Población } \\
\text { hipocalórica }\end{array}$ & $\begin{array}{c}\text { Método } \\
\text { valoración } \\
\text { composición } \\
\text { corporal }\end{array}$ & Dieta cetogénica & Dieta hipocalórica & Co intervenciones \\
\hline $\begin{array}{l}\text { Hussain y } \\
\text { colaboradores } \\
(2012)^{(27)} \\
\text { Kuwait }\end{array}$ & $\begin{array}{l}\text { Controlado } \\
\text { no } \\
\text { aleatorizado } \\
6\end{array}$ & $\begin{array}{l}\text { H y M } 142 \\
36,8 \pm 0,6 \text { años } \\
\text { IMC } 37,2 \pm 5,7 \\
\mathrm{~kg} / \mathrm{m}^{2}\end{array}$ & $\begin{array}{l}\text { H y M } 119 \\
34,8 \pm 0,7 \text { años } \\
\mathrm{IMC} 36,0 \pm 6,1 \\
\mathrm{~kg} / \mathrm{m}^{2}\end{array}$ & Antropometría & $\begin{array}{l}\text { Dieta cetogénica muy baja en } \\
\text { calorías }\end{array}$ & $\begin{array}{l}\text { Dieta baja en calorías } \\
2200 \mathrm{kcal} / \text { día }\end{array}$ & $\begin{array}{l}\text {-24 visitas para consejería } \\
\text { en dieta y medicamentos } \\
\text {-Tableta estándar } \\
\text { multivitamínica } \\
\text { multimineral } \\
\text {-Tomar } 6 \text { a } 8 \text { vasos de agua } \\
\text { diariamente }\end{array}$ \\
\hline $\begin{array}{l}\text { Westman y } \\
\text { colaboradores } \\
(2008)^{(28)} \\
\text { Estados } \\
\text { Unidos }\end{array}$ & $\begin{array}{l}\text { Controlado } \\
\text { aleatorizado } \\
6\end{array}$ & $\begin{array}{l}\text { H } 7 \\
\text { M } 14 \\
51,2 \pm 6,1 \text { años } \\
\text { IMC } 37,8 \pm 6,7 \\
\mathrm{~kg} / \mathrm{m}^{2}\end{array}$ & $\begin{array}{l}\text { H } 6 \\
\text { M } 23 \\
50 \pm 8,4 \text { años IMC } \\
37,9 \pm 6,0 \mathrm{~kg} / \mathrm{m}^{2}\end{array}$ & Antropometría & $\begin{array}{l}\text { No se hizo restricción calórica }<20 \mathrm{~g} \\
\text { carbohidratos/día }\end{array}$ & $\begin{array}{l}\text { Dieta de bajo índice } \\
\text { glucémico } \\
-500 \text { kcal } \\
55 \% \text { carbohidratos }\end{array}$ & $\begin{array}{l}\text {-13 visitas -Suplementación } \\
\text { nutricional -Motivados a } \\
\text { ejercitarse por } 30 \text { minutos, } \\
3 \text { veces a la semana y sin } \\
\text { programa de ejercicio. } \\
\text {-Tomar al menos } 6 \text { vasos } \\
\text { de las bebidas permitidas } \\
\text { diariamente }\end{array}$ \\
\hline $\begin{array}{l}\text { Johnstone y } \\
\text { colaboradores } \\
(2006)^{(29)} \\
\text { Estados } \\
\text { Unidos } \\
\end{array}$ & $\begin{array}{l}\text { Controlado } \\
\text { aleatorizado } \\
1,5\end{array}$ & $\begin{array}{l}\mathrm{H} 2 \\
\mathrm{M} 7 \\
38,4 \pm 3,9 \text { años } \\
\mathrm{IMC} 34,9 \pm 1,6 \\
\mathrm{~kg} / \mathrm{m}^{2}\end{array}$ & $\begin{array}{l}\mathrm{H} 2 \\
\text { M } 8 \\
37,2 \pm 3,9 \text { años } \\
\mathrm{IMC} 34,6 \pm 1,5 \\
\mathrm{~kg} / \mathrm{m}^{2}\end{array}$ & BIA & $\begin{array}{l}1500 \mathrm{kcal} / \mathrm{día} \\
60 \% \text { grasas } \\
10 \% \text { carbohidratos } \\
30 \% \text { proteína }\end{array}$ & $\begin{array}{l}1500 \text { kcal/día } \\
30 \% \text { grasas } \\
40 \% \text { carbohidratos } \\
30 \% \text { proteína }\end{array}$ & $\begin{array}{l}\text { Las comidas y bebidas } \\
\text { fueron suministradas a los } \\
\text { participantes } \\
\text { Se mantuvieron } \\
\text { sedentarios }\end{array}$ \\
\hline $\begin{array}{l}\text { Yancy Jr. Y } \\
\text { colaboradores } \\
(2004)^{(30)} \\
\text { Estado Unidos }\end{array}$ & $\begin{array}{l}\text { Controlado } \\
\text { aleatorizado } \\
6\end{array}$ & $\begin{array}{l}\text { H } 13 \\
\text { M } 32 \\
45,3 \pm 9,5 \text { años } \\
\text { IMC } 34,6 \pm 5,2\end{array}$ & $\begin{array}{l}\text { H } 8 \\
\text { M } 26 \\
44,1 \pm 8,7 \text { años } \\
\text { IMC } 33,9 \pm 5,3\end{array}$ & BIA & $\begin{array}{l}68 \% \text { lípidos } \\
29 \pm 11,1 \mathrm{~g} \text { carbohidratos/día (8 } \\
\% \mathrm{kcal} \text { ) } \\
26 \% \text { proteína }\end{array}$ & $\begin{array}{l}-500 \text { a -1000 kcal /día } \\
29 \% \text { lípidos } \\
198 \pm 34,2 \text { g carbohidratos } \\
(52 \%) \\
19 \% \text { proteína }\end{array}$ & $\begin{array}{l}\text {-9 reuniones grupales } \\
\text {-Recomendaciones para } \\
\text { ejercicio durante } 30 \\
\text { minutos diarios al menos } 3 \\
\text { veces a la semana. } \\
\text {-Tomar de } 6 \text { a } 8 \text { vasos de } \\
\text { agua diarios } \\
\text {-Suplemento nutricional }\end{array}$ \\
\hline
\end{tabular}

GCT: gasto calórico total. 
Tabla 2. Evaluación del riesgo de sesgo en los estudios incluidos

\begin{tabular}{|l|l|l|l|l|l|l|l|}
\hline \multicolumn{1}{|c|}{ Artículos incluidos } & $\begin{array}{c}\text { Proceso de } \\
\text { aleatorización }\end{array}$ & $\begin{array}{c}\text { Enmascaramiento } \\
\text { paciente }\end{array}$ & $\begin{array}{c}\text { Adherencia } \\
\text { a la } \\
\text { intervención }\end{array}$ & $\begin{array}{c}\text { Datos } \\
\text { incompletos }\end{array}$ & $\begin{array}{c}\text { Medición } \\
\text { del } \\
\text { desenlace }\end{array}$ & $\begin{array}{c}\text { Sesgo de } \\
\text { reporte } \\
\text { selectivo }\end{array}$ & $\begin{array}{c}\text { Evaluación } \\
\text { global riesgo } \\
\text { de sesgo }\end{array}$ \\
\hline $\begin{array}{l}\text { Perticone y colaboradores } \\
(2019)^{(22)}\end{array}$ & & & & & \\
\hline $\begin{array}{l}\text { Sajoux y colaboradores } \\
(2019)^{(23)}\end{array}$ & & & & & \\
\hline $\begin{array}{l}\text { Saslow y colaboradores } \\
(2017)^{(24)}\end{array}$ & & & & & \\
\hline $\begin{array}{l}\text { Moreno y colaboradores } \\
(2016)^{(18)}\end{array}$ & & & & & & \\
\hline $\begin{array}{l}\text { Goday y colaboradores } \\
(2016)^{(25)}\end{array}$ & & & & & & \\
\hline $\begin{array}{l}\text { Rubini y colaboradores } \\
(2015)^{(26)}\end{array}$ & & & & & & \\
\hline $\begin{array}{l}\text { Hussain y colaboradores } \\
(2012)^{(27)}\end{array}$ & & & & & & \\
\hline $\begin{array}{l}\text { Westman y colaboradores } \\
(2008)^{(28)}\end{array}$ & & & & & \\
\hline $\begin{array}{l}\text { Johnstone y } \\
\text { colaboradores (2006) }\end{array}$ & & & & & \\
\hline $\begin{array}{l}\text { Yancy Jr.Y colaboradores } \\
(2004)^{(30)}\end{array}$ & & & & & \\
\hline
\end{tabular}

Blanco: bajo riesgo de sesgo; gris: posible riesgo de sesgo; negro: alto riesgo de sesgo.

que la dieta hipocalórica, sin importar la diferencia de restricción calórica entre las dietas (Figura 3).

\section{Cambios del índice de masa corporal}

El IMC se reportó en 6 investigaciones, de las cuales 3 indicaron una mayor pérdida de IMC en los pacientes que siguieron la dieta cetogénica. El metaanálisis global indica que la dieta cetogénica podría reducir el IMC en $1,6 \mathrm{~kg} / \mathrm{m}^{2}$ más (IC del $95 \% 3,0$ a 0,15 ) que con la dieta hipocalórica; dichos efectos se incrementan con la realización de ejercicio y períodos de más de 6 meses (Figura 4). En cuanto a la restricción calórica, el metaanálisis evidencia que el cambio de IMC debido a la dieta cetogénica no se altera por la diferencia de restricción calórica entre las dietas (Figura 5).

\section{Cambios de masa grasa}

De los 6 artículos que utilizaron DXA o BIA para medir la composición corporal, únicamente 4 reportaron valores de masa grasa; de estos, 3 mostraron diferencias significativas entre la dieta cetogénica y la dieta hipocalórica. El metaanálisis global reveló que la dieta cetogénica tiene una mayor pérdida de masa grasa que la dieta hipocalórica $(-0,79 \mathrm{~kg}$ IC del $95 \%-1,1$ a $-0,5)$ y no hay diferencia si se realiza con o sin ejercicio (Figura 6).

\section{Cambios de masa libre de grasa}

La masa libre de grasa fue reportada por 4 de los 6 artículos, en donde se encontraron valores diferentes entre los estudios. El metaanálisis global indica que no hay una diferencia entre los cambios de masa libre de grasa entre la dieta cetogénica y la dieta hipocalórica, pero la realización de ejercicio con la dieta cetogénica podría incrementar la pérdida de masa libre de grasa (Figura 7). Por otra parte, únicamente el estudio de Perticone y colaboradores ${ }^{(22)}$ reportó valores de masa muscular, lo cual describe que presuntamente no hay cambios en la masa muscular al comparar las dos intervenciones. 


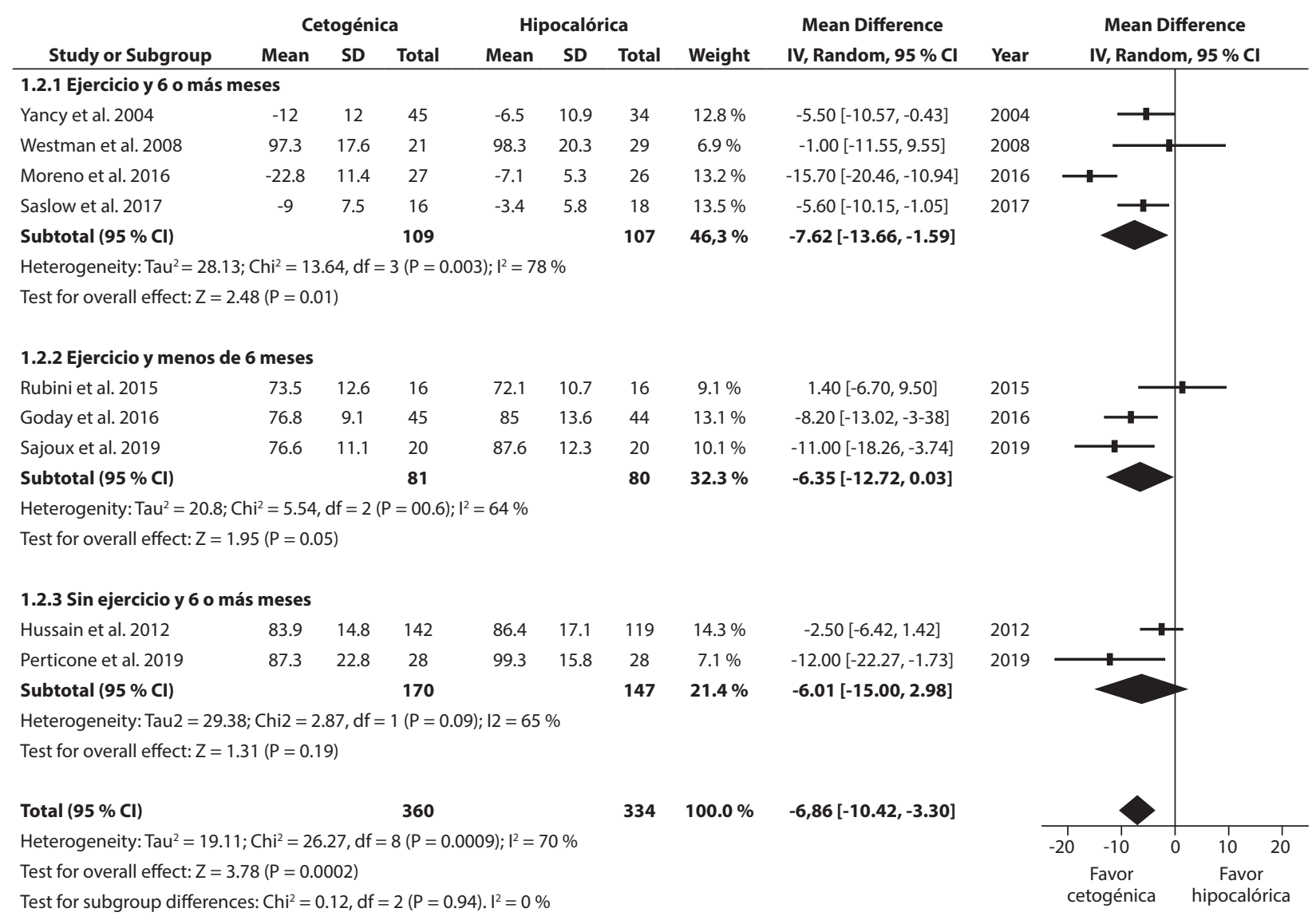

Figura 2. Gráfico de bosque de la diferencia de cambio de peso entre la dieta cetogénica y la dieta hipocalórica, según la duración del estudio y la realización de ejercicio.

Por último, los gráficos de embudo describen que posiblemente existe un sesgo de información en los resultados de peso e IMC; sin embargo, no se pudo evaluar la simetría por métodos estadísticos (prueba de Egger) debido a la inclusión de menos de 10 artículos (Figura 8).

\section{DISCUSIÓN}

La presente revisión sistemática y metaanálisis compara los efectos de la dieta cetogénica baja en calorías con la dieta hipocalórica sobre los cambios de peso y composición corporal en adultos con exceso de peso. Es el primer metaanálisis en el que se muestra que la dieta cetogénica y la dieta hipocalórica no presentan diferencias en cuanto a la pérdida de masa magra, y la dieta cetogénica genera una mayor reducción de peso, IMC y masa grasa. Sin embargo, el bajo riesgo de sesgo es inusual en los estudios incluidos y posiblemente existe un sesgo de publicación, por lo que el efecto real de la dieta cetogénica comparada con la dieta hipocalórica podría estar sobreestimado.

En cuanto al riesgo de sesgo (Tabla 2), los estudios reportaban el seguimiento a los pacientes, pero el reporte de ingesta de nutrientes es escaso, y la concentración de cuerpos cetónicos fue nulo. Con respecto a la medición del desenlace y el reporte selectivo, hay heterogeneidad en el informe de valores, donde algunos indican diferencias finales, valores finales o un valor $p$. Futuros estudios deberían preferir diferencias finales y/o valores finales junto a un valor $p$, lo cual facilitará su inclusión en un metaanálisis y permitirá al lector hacer una apreciación objetiva del efecto real de la intervención.

Como se observa en todas las gráficas de bosques, los resultados en cada desenlace son heterogéneos, lo cual se puede deber a la heterogeneidad en la metodo- 


\begin{tabular}{|c|c|c|c|c|c|c|c|c|c|c|}
\hline \multirow[b]{2}{*}{ Study or Subgroup } & \multicolumn{3}{|c|}{ Cetogénica } & \multicolumn{3}{|c|}{ Hipocalórica } & \multirow[b]{2}{*}{ Weight } & \multirow{2}{*}{$\begin{array}{c}\text { Mean Difference } \\
\text { IV, Random, } 95 \% \mathrm{Cl}\end{array}$} & \multirow{2}{*}{\multicolumn{2}{|c|}{$\begin{array}{c}\text { Mean Difference } \\
\text { IV, Random, } 95 \% \mathrm{Cl}\end{array}$}} \\
\hline & Mean [Kg] & SD [Kg] & Total & Mean $[\mathrm{Kg}]$ & SD [Kg] & Total & & & & \\
\hline \multicolumn{11}{|c|}{ 1.3.2 Mayor en la cetogénica } \\
\hline Goday et al. 2016 & 76.8 & 9.1 & 45 & 85 & 13.6 & 44 & $13.1 \%$ & $-8.20[-13.02,-3.38]$ & & \\
\hline Hussain et al. 2012 & 83.9 & 14.8 & 142 & 86.4 & 17.1 & 119 & $14.3 \%$ & $-2.50[-6.42,1.42]$ & & + \\
\hline Moreno et al. 2016 & -22.8 & 11.4 & 27 & -7.1 & 5.3 & 26 & $13.2 \%$ & $-15.70[-20.46,-10.94]$ & $\longrightarrow$ & \\
\hline Perticone et al. 2019 & 87.3 & 22.8 & 28 & 99.3 & 15.8 & 28 & $7.1 \%$ & $-12.00[-22-27,-1.73]$ & & \\
\hline Rubini et al. 2015 & 73.5 & 12.6 & 16 & 72.1 & 10.7 & 16 & $9.1 \%$ & $1.40[-6.70,9.50]$ & & 上 \\
\hline Sajoux et al. 2019 & 76.6 & 11.1 & 20 & 87.6 & 12.3 & 20 & $10.1 \%$ & $-11.00[-18.26,-3.74]$ & & \\
\hline Subtotal (95 \% Cl) & & & 278 & & & 253 & $66.9 \%$ & $-7.98[-13.26,-2.69]$ & & \\
\hline \multicolumn{11}{|c|}{ Heterogeneity: $\mathrm{Tau}^{2}=32.61 ; \mathrm{Chi}^{2}=23.98, \mathrm{df}=5(\mathrm{P}=0.0002) ; \mathrm{I}^{2}=79 \%$} \\
\hline \multicolumn{11}{|c|}{ Test overall effect: $Z=2.96(P=0.003)$} \\
\hline \multicolumn{11}{|c|}{ 1.3.3 Mayor en la hipocalórica } \\
\hline Saslow et al. 2017 & -9 & 7.5 & 16 & -3.4 & 5.8 & 18 & $13.5 \%$ & $-5.60[-10.15,-1.05]$ & & \\
\hline Westman et al. 2008 & 97.3 & 17.6 & 21 & 98.3 & 20.3 & 29 & $6.9 \%$ & $-1.00[-11.55,9.55]$ & & \\
\hline Yancy Jr et al. 2004 & -12 & 12 & 45 & -6.5 & 10.9 & 34 & $12.8 \%$ & $-5.50[-10.57,-0.43]$ & & \\
\hline Subtotal $(95 \% \mathrm{Cl})$ & & & 82 & & & 81 & $33.1 \%$ & $-5.13[-8.35,-1.91]$ & & \\
\hline \multicolumn{11}{|c|}{ Heterogeneity: $\mathrm{Tau}^{2}=0.00 ; \mathrm{Chi}^{2}=0.65, \mathrm{df}=2(\mathrm{P}=0.72) ; 12=0 \%$} \\
\hline \multicolumn{11}{|c|}{ Test for overall effect: $Z=3.12(P=0.002)$} \\
\hline Total $(95 \% \mathrm{Cl})$ & & & 360 & & & 334 & $100.0 \%$ & $-6.86[-10.42,3.30]$ & & \\
\hline \multicolumn{9}{|c|}{ Heterogeneity: $\mathrm{Tau}^{2}=19.11 ; \mathrm{Chi}^{2}=26.27, \mathrm{df}=8(\mathrm{P}=0.0009) ; \mathrm{I}^{2}=70 \%$} & -20 & 10 \\
\hline \multicolumn{9}{|c|}{ Test for overall effect: $Z=3.78(P=0.0002)$} & $\begin{array}{l}\text { Favor } \\
\text { togénica }\end{array}$ & $\begin{array}{l}\text { Favor } \\
\text { hipalórica }\end{array}$ \\
\hline Test for subgroup differe & ces: $\mathrm{Chi}^{2}=0.8$ & $1, d f=1$ & $=0.37)$, & $=0 \%$ & & & & & génica & hipocalórica \\
\hline
\end{tabular}

Figura 3. Gráfico de bosque de la diferencia de cambio de peso entre la dieta cetogénica y la dieta hipocalórica según la restricción calórica.

logía de los estudios, principalmente en la inclusión de hombres y/o mujeres, la duración y la definición de cada intervención. Sin embargo, los valores globales indican que la dieta cetogénica tiene mayores efectos que la dieta hipocalórica sobre el peso, el IMC y la masa grasa, como se discute a continuación.

En cuanto a la pérdida de peso corporal, los resultados de este metaanálisis indican que la dieta cetogénica promueve una mayor pérdida de peso que la dieta hipocalórica, lo que coincide con metaanálisis previos elaborados con modelos frecuentistas y bayesianos; sin embargo, a pesar de que los resultados de este y anteriores metaanálisis están orientados en la misma dirección, se observan discrepancias entre los valores agregados de cada metaanálisis, lo cual se puede atribuir a las diferencias en los criterios de selección e intervenciones entre los estudios primarios de cada uno de estos. Un ejemplo de esto es el uso de cointervenciones para los cambios de estilos de vida, pacientes con diabetes, períodos de seguimiento, distribución de sexo, gradientes de edad, definición de dieta cetogénica e intervención de control ${ }^{(1,2,11}$, ${ }^{31-35)}$. Por lo tanto, futuros experimentos y metaanálisis deberán tender hacia el establecimiento de criterios de selección amplios y definidos que permitan realizar análisis por subgrupos, como un grupo de diabéticos, obesos y con actividad física.

Respecto a la restricción calórica y el peso corporal, los resultados indican que hay cambios en el peso corporal debido a la reducción calórica (Figura 3), lo que significa que a largo plazo la composición de macronutrientes no determina una mayor pérdida de peso ${ }^{(20,}$ ${ }^{31,33)}$. Por tanto, se puede inferir que para la pérdida de peso corporal a corto plazo, la reducción calórica y la composición de macronutrientes son importantes, pero a largo plazo no se requieren modificaciones en la composición de nutrientes; es la reducción calórica la que mantendrá la pérdida de peso ${ }^{(32)}$.

Estudios previos han indicado que en seguimientos de más de 12 meses, la diferencia de peso corporal entre ambos planes de alimentación es menor a $1 \mathrm{~kg}$ o incluso no se presentan diferencias significativas, lo cual se debe a que la fase final de una dieta cetogénica contempla una dieta isocalórica ${ }^{(2,20,34)}$. Por esta razón, se puede afirmar que la dieta cetogénica podría emplearse 


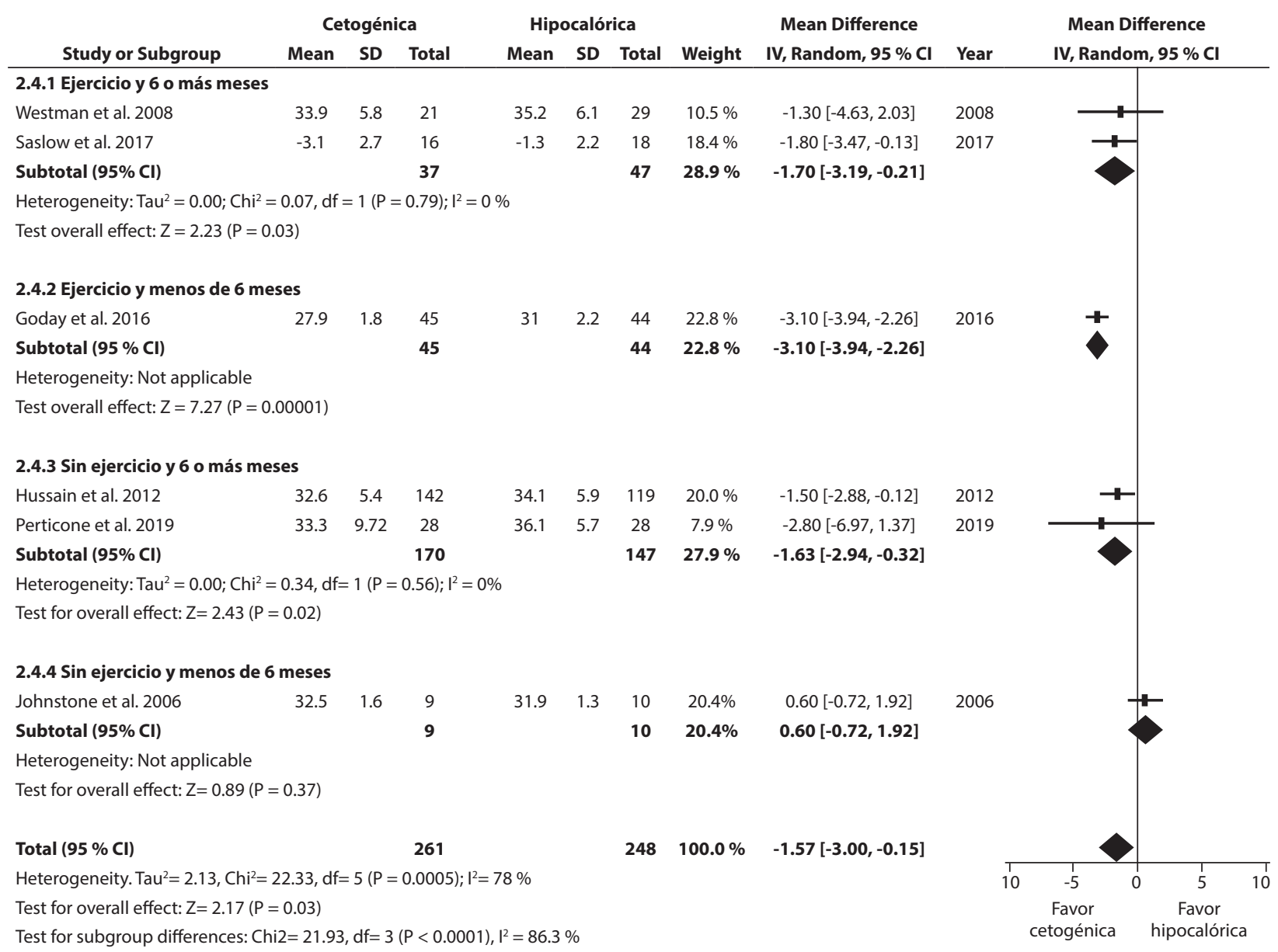

Figura 4. Gráfico de bosque de la diferencia de cambio del IMC entre la dieta cetogénica y la dieta hipocalórica según la duración del estudio y la realización de ejercicio.

hasta por un año, tiempo durante el cual su fase final debe enfocarse fuertemente en la educación nutricional que permita llegar y mantener un peso saludable, al tiempo que promueva hábitos alimentarios saludables para el resto de la vida ${ }^{(1)}$.

Solo 6 de los 10 artículos informaron los cambios en el IMC, y el metaanálisis encontró que la dieta cetogénica genera una pequeña reducción de $1,6 \mathrm{~kg} / \mathrm{m}^{2}$ más (IC del $95 \%$ 3,0 a 0,15) que la dieta hipocalórica, lo cual difiere a lo encontrado por Choi y colaboradores ${ }^{(36)}$ $\left(-0,63 \mathrm{~kg} / \mathrm{m}^{2}\right.$ IC del $95 \%-1,35$ a 0,08$)$ en personas con y sin diabetes, de manera similar a lo reportado por Castellana y colaboradores $^{(1)}\left(-6,2 \mathrm{~kg} / \mathrm{m}^{2}\right.$ IC del $95 \%$ $-7,4$ a -4,9) en estudios de al menos 4 semanas de seguimiento. El hecho de que Choi y colaboradores ${ }^{(36)}$ estuvieron cerca de la significancia estadística y los hallazgos del presente metaanálisis sugieren cambios pequeños del IMC indica que la discrepancia entre los metaanálisis podría deberse a la falta de reporte de cambios en el IMC en los estudios primarios y a un posible sesgo de reporte relacionado con la omisión de resultados no significativos en el IMC.

Respecto a la masa grasa, el metaanálisis indica una reducción de -0,8 kg (IC del $95 \%-1,1$ a -0,5), lo cual es pequeño pero en el mismo sentido a lo reportado por Castellana y colaboradores ${ }^{(1)}(-4,4 \mathrm{~kg}$ IC del $95 \%$ $-5,3$ a -3,6). La diferencia en la precisión del intervalo de confianza se puede deber a que el metaanálisis de Castellana y colaboradores ${ }^{(1)}$ involucró estudios con menos de 4 semanas de seguimiento (tiempo en el cual se observa un mayor efecto de la dieta cetogénica) y pacientes programados para una cirugía bariátrica (quienes requieren una pérdida rápida de grasa previa a la cirugía); sin embargo, en el presente metaanálisis 


\begin{tabular}{|c|c|c|c|c|c|c|c|c|c|c|c|}
\hline \multirow[b]{2}{*}{ Study or Subgroup } & \multicolumn{3}{|c|}{ Cetogénica } & \multicolumn{3}{|c|}{ Hipocalórica } & \multicolumn{3}{|c|}{ Mean Difference } & \multirow{2}{*}{\multicolumn{2}{|c|}{$\begin{array}{c}\text { Mean Difference } \\
\text { IV, Random, } 95 \% \mathrm{Cl}\end{array}$}} \\
\hline & $\begin{array}{c}\text { Mean } \\
{\left[\mathbf{k g} / \mathbf{m}^{2}\right]}\end{array}$ & $\begin{array}{c}\text { SD } \\
{\left[\mathbf{k g} / \mathbf{m}^{2}\right]}\end{array}$ & Total & $\begin{array}{c}\text { Mean } \\
{\left[\mathrm{kg} / \mathrm{m}^{2}\right]}\end{array}$ & $\begin{array}{c}\text { SD } \\
{\left[\mathbf{k g} / \mathrm{m}^{2}\right]}\end{array}$ & Total & Weight & IV, Random, $95 \%$ CI & Year & & \\
\hline \multicolumn{12}{|c|}{ 2.5.1 Mayor en la cetogénica } \\
\hline Hussain et al. 2012 & 32.6 & 5.4 & 142 & 34.1 & 5.9 & 119 & $20.0 \%$ & $-1.50[-2.88,-0.12]$ & 2012 & 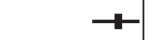 & \\
\hline Goday et al. 2016 & 27.9 & 1.8 & 45 & 31 & 2.2 & 44 & $22.8 \%$ & $-3.10[-3.94,-2.26]$ & 2016 & & \\
\hline Perticone et al. 2019 & 33.3 & 9.7 & 28 & 36.1 & 5.7 & 28 & $7.9 \%$ & $-2.80[-6.97,1.37]$ & 2019 & & \\
\hline Subtotal $(95 \% \mathrm{Cl})$ & & & 215 & & & 191 & $50.7 \%$ & $-2.47[-3.70,-1.24]$ & & & \\
\hline \multicolumn{12}{|c|}{ Heterogeneity: $\mathrm{Tau}^{2}=0.54 ; \mathrm{Chi}^{2}=3.77, \mathrm{df}=2(\mathrm{P}=0.15) ; \mathrm{l}^{2}=47 \%$} \\
\hline \multicolumn{12}{|c|}{ Test for overall effect $Z=3.95(P<0.0001)$} \\
\hline \multicolumn{12}{|c|}{ 2.5.2 Mayor en la hipocalórica } \\
\hline Johnstone et al. 2006 & 32.5 & 1.6 & 9 & 31.9 & 1.3 & 10 & 20.4 & $0.60[-0.72,1.92]$ & 2006 & & \\
\hline Westman et al. 2008 & 33.9 & 5.8 & 21 & 35.2 & 6.1 & 29 & $10.5 \%$ & $-1.30[-4.63,2.03]$ & 2008 & & \\
\hline Saslow et al. 2017 & -3.1 & 2.7 & 16 & -1.3 & 2.2 & 18 & $18.4 \%$ & $-1.80[-3.47,-0.13]$ & 2017 & & \\
\hline Subtotal $(95 \% \mathrm{Cl})$ & & & 46 & & & 57 & $49.3 \%$ & $-0.67[-2.45,1.11]$ & & & \\
\hline \multicolumn{12}{|c|}{ Heterogeneity: $\mathrm{Tau}^{2}=1.46 ; \mathrm{Chi}^{2}=5.19, \mathrm{df}=2(\mathrm{P}=0.07) ; \mathrm{I}^{2}=61 \%$} \\
\hline \multicolumn{12}{|c|}{ Test for overall effect $Z=0.74(P=0.46)$} \\
\hline Total $(95 \% \mathrm{Cl})$ & & & 261 & & & 248 & $100.0 \%$ & $-1.57[-3.00,-0,15]$ & & & \\
\hline \multicolumn{6}{|c|}{ Heterogeneity: $\mathrm{Tau}^{2}=2.13 ; \mathrm{Chi}^{2}=22.33, \mathrm{df}=5(\mathrm{P}=0.0005) ; \mathrm{I}^{2}=78 \%$} & & & & 10 & $\begin{array}{l}1 \\
-5\end{array}$ & 5 \\
\hline \multicolumn{6}{|c|}{ Test for overall effect $Z=2.17(P=0.03)$} & & & & & Favor & Favor \\
\hline Test for subgroup differ & ences: $\mathrm{Chi}^{2}$ & $=2.66$ & $=1,(\mathrm{P}$ & 17 & & & & & & & \\
\hline
\end{tabular}

Figura 5. Gráfico de bosque de la diferencia de cambio de IMC entre la dieta cetogénica y la dieta hipocalórica según la restricción calórica.

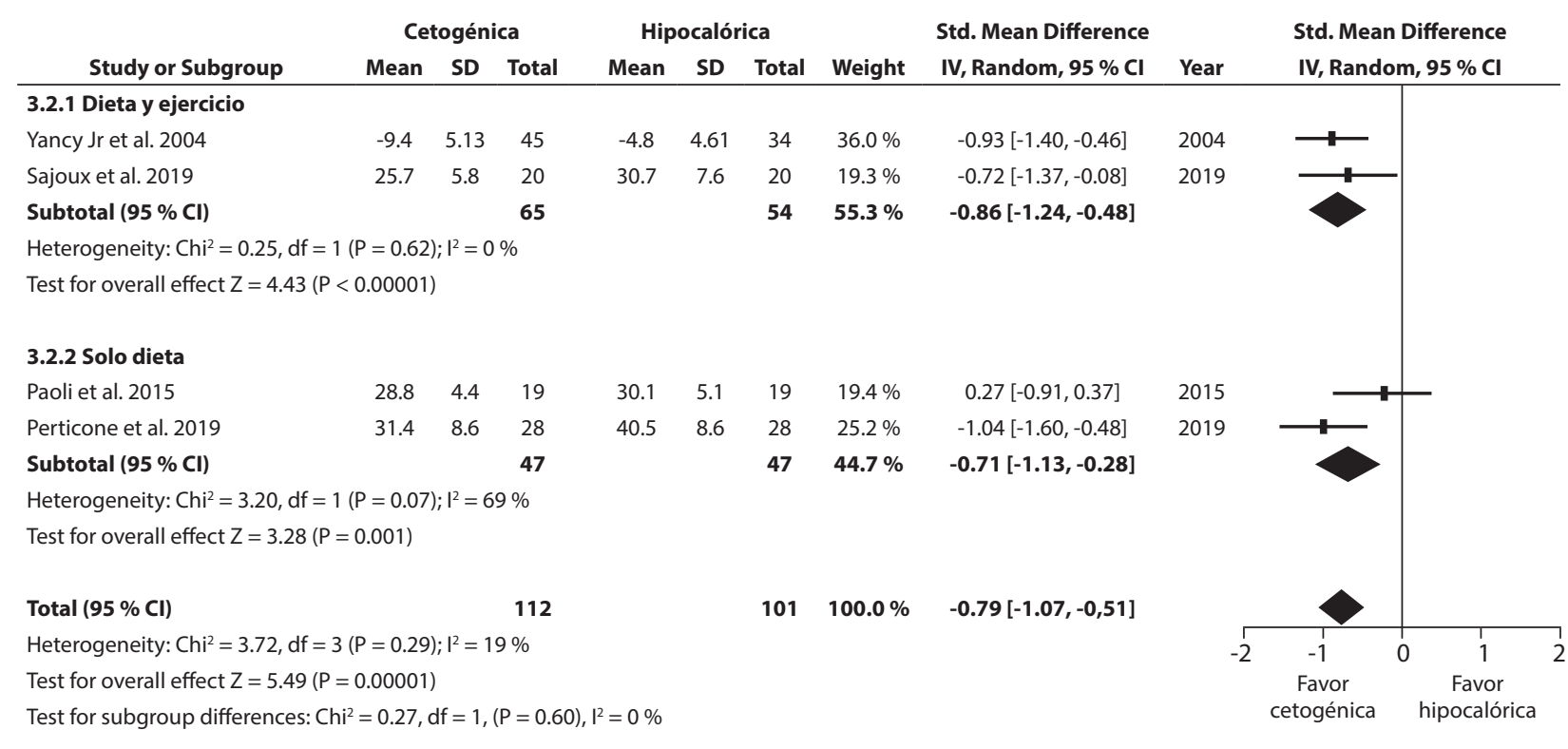

Figura 6. Gráfico de bosque de la diferencia del cambio de masa grasa entre la dieta cetogénica y la dieta hipocalórica según la realización de ejercicio. 


\begin{tabular}{|c|c|c|c|c|c|c|c|c|c|c|}
\hline \multirow[b]{2}{*}{ Study or Subgroup } & \multicolumn{3}{|c|}{ Cetogénica } & \multicolumn{3}{|c|}{ Hipocalórica } & \multicolumn{3}{|c|}{ Std. Mean Difference } & \multirow{2}{*}{$\begin{array}{l}\text { Std. Mean Difference } \\
\text { IV, Random, } 95 \% \mathrm{CI}\end{array}$} \\
\hline & Mean & SD & Total & Mean & SD & Total & Weight & IV, Random, $95 \% \mathrm{Cl}$ & Year & \\
\hline \multicolumn{11}{|l|}{ 4.2.1 Dieta y ejercicio } \\
\hline Yancy Jr et al. 2004 & -3.3 & 2.05 & 45 & -2.4 & 2.08 & 34 & $29.2 \%$ & $-0.43[-0.88,-0.02]$ & 2004 & \\
\hline Sajoux et al. 2019 & 49.1 & 9.7 & 20 & 56.9 & 11.2 & 20 & $24.7 \%$ & $-0.73[-1.37,-0.09]$ & 2019 & \\
\hline Subtotal $(95 \% \mathrm{Cl})$ & & & 65 & & & 54 & $53.8 \%$ & $-0.53[-0.90,-0.16]$ & & \\
\hline \multicolumn{11}{|c|}{ Heterogeneity: $\operatorname{Tau}^{2}=0.00 ; \mathrm{Chi}^{2}=0.55, \mathrm{df}=1(\mathrm{P}=0.46) ; \mathrm{I}^{2}=0 \%$} \\
\hline \multicolumn{11}{|c|}{ Test for overall effect $Z=2.82(P<0.005)$} \\
\hline \multicolumn{11}{|l|}{ 4.2.2 Solo dieta } \\
\hline Johnstone et al. 2006 & 53.2 & 3.9 & 9 & 54.3 & 3.4 & 10 & $18.9 \%$ & $0.29[-1.19,0.62]$ & 2006 & \\
\hline Perticone et al. 2019 & 62.7 & 15.6 & 28 & 55.3 & 11.5 & 28 & $27.2 \%$ & $0.53[-0.00,1.07]$ & 2019 & \\
\hline Subtotal $(95 \% \mathrm{Cl})$ & & & 37 & & & 38 & $46.2 \%$ & $0.21[-0.58,0.99]$ & & \\
\hline \multicolumn{11}{|c|}{ Heterogeneity: $\mathrm{Tau}^{2}=0.19 ; \mathrm{Chi}^{2}=2.34, \mathrm{df}=1(\mathrm{P}=0.13) ; 1^{2}=57 \%$} \\
\hline \multicolumn{11}{|c|}{ Test for overall effect $Z=0.52(P=0.61)$} \\
\hline Total $(95 \% \mathrm{Cl})$ & & & 102 & & & 92 & $100.0 \%$ & $-0.22[-0.79,0,36]$ & & \\
\hline \multicolumn{9}{|c|}{ Heterogeneity: $\mathrm{Tau}^{2}=0.24 ; \mathrm{Chi}^{2}=10.90, \mathrm{df}=3(\mathrm{P}=0.01) ; \mathrm{I}^{2}=72 \%$} & -2 & $\begin{array}{ll}1 \\
-1\end{array}$ \\
\hline \multicolumn{9}{|c|}{ Test for overall effect $Z=0.73(P=0.46)$} & \multirow{2}{*}{\multicolumn{2}{|c|}{$\begin{array}{cc}\begin{array}{c}\text { Favor } \\
\text { cetogénica }\end{array} & \begin{array}{c}\text { Favor } \\
\text { hipocalórica }\end{array}\end{array}$}} \\
\hline Test for subgroup differen & $\mathrm{Chi}^{2}=2.2$ & $7, \mathrm{df}=$ & $1,(P=0$. & $=6$ & & & & & & \\
\hline
\end{tabular}

Figura 7. Gráfico de bosque de la diferencia del cambio de masa libre de grasa entre la dieta cetogénica y la dieta hipocalórica según la realización de ejercicio.

\section{Cambio de peso}

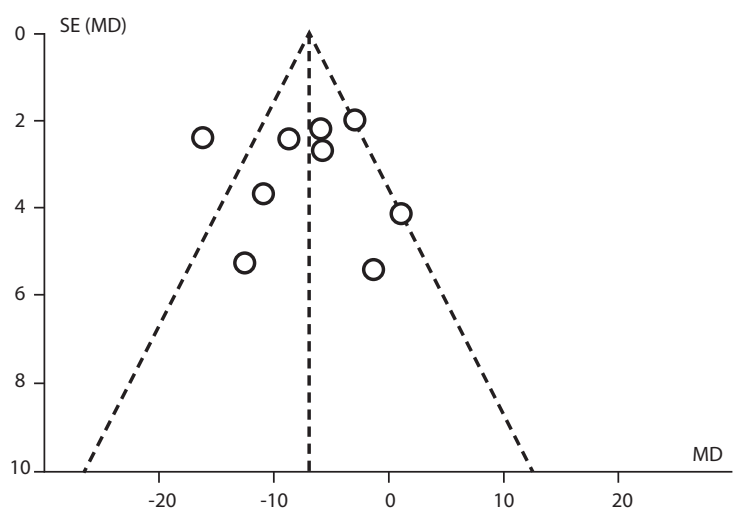

Cambio de masa magra

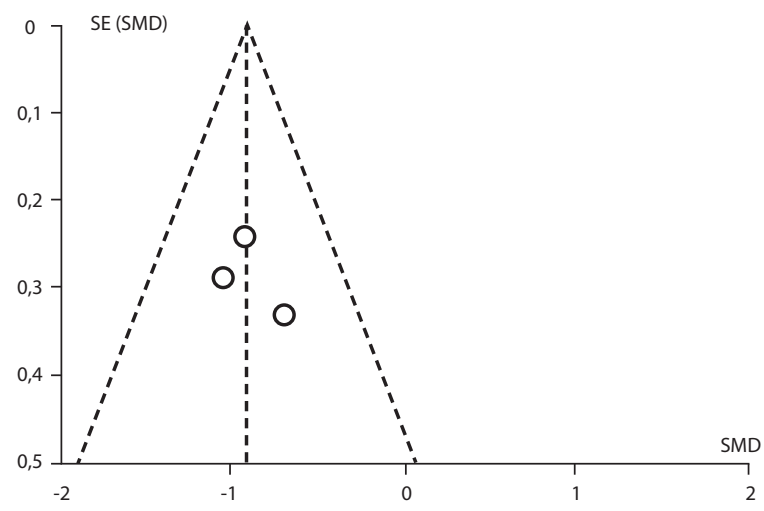

\section{Cambio de IMC}

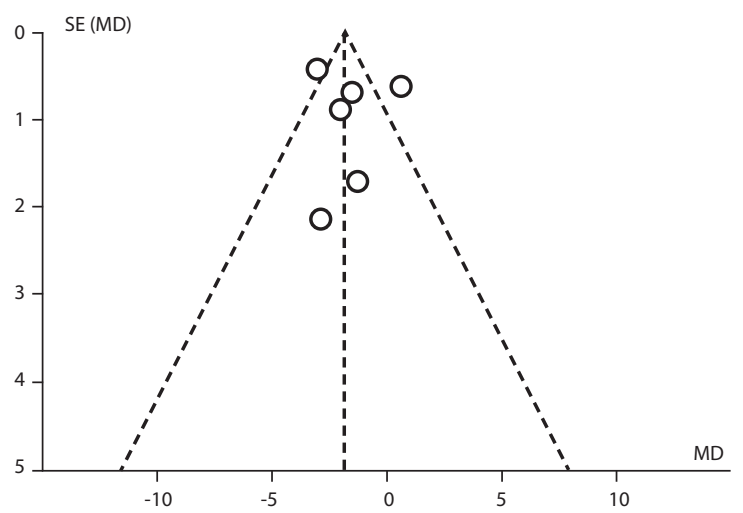

Cambio de masa libre de grasa

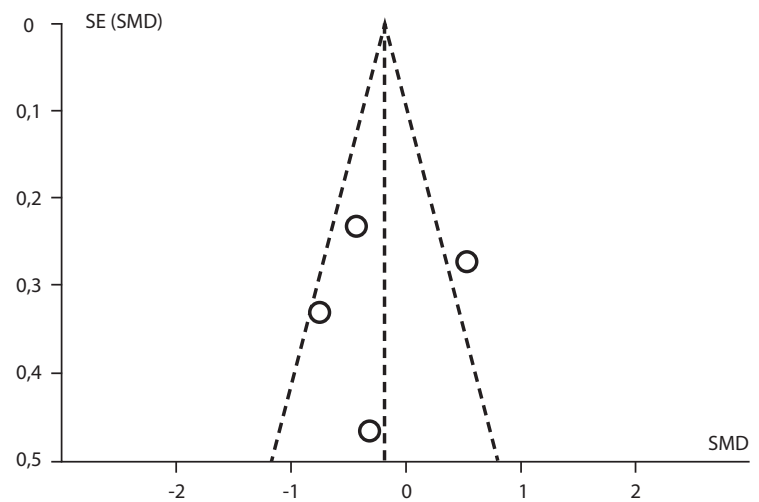

Figura 8. Gráficos de embudo para los desenlaces de cambio de peso, IMC, masa grasa y masa libre de grasa. MD: mean differences; SE: standard error; SMD: standardized mean difference. 
la información de masa grasa solo fue reportada por 3 de los 6 artículos con una medición de la composición corporal. Por lo tanto, hay que considerar la posible existencia de un sesgo de omisión de información que podría estar sobrestimando el efecto de la dieta cetogénica en la masa grasa.

El metaanálisis describió que la masa magra o la masa libre de grasa no difiere entre la dieta cetogénica y la dieta hipocalórica $(-0,22 \mathrm{~kg}$ IC del $95 \%-0,8$ a 0,4); no obstante, en el análisis de ejercicio con dieta cetogénica (Figura 7) se observaron pérdidas de masa magra de $-0,5 \mathrm{~kg}$ (IC del $95 \%-0,9$ a -0,2), lo cual es inferior y es similar a lo reportado por Castellana y colaboradores $^{(1)}(-4,4 \mathrm{~kg}$ IC del 95\% -5,3 a -3,6). La desigualdad entre los metaanálisis podría atribuirse a los cambios de composición entre las fases de la dieta cetogénica. Metaanálisis que involucren estudios de menos de 4 semanas $^{(1)}$ abarcan la fase inicial de la dieta cetogénica, la cual moviliza macronutrientes para la gluconeogénesis y la elaboración de cuerpos cetónicos generan grandes pérdidas de masa muscular. Metaanálisis de más de 4 semanas, como el presente estudio, describen un bajo impacto sobre la masa magra y podría ser un efecto residual de la primera fase de la dieta cetogénica.

Este metaanálisis no evaluó el efecto de la dieta cetogénica sobre la masa muscular esquelética debido a que únicamente el estudio de Perticone y colaboradores $^{(22)}$ describe que presuntamente no hay cambios en la masa muscular al comparar las dos intervenciones. El metaanálisis de Lee ${ }^{(19)}$ indica que la dieta cetogénica con ejercicio y una adecuada suplementación de proteína podría conservar la masa libre de grasa, pero realmente se desconoce el impacto de la dieta cetogénica sobre la masa muscular. Por ejemplo, en los estudios incluidos en el presente metaanálisis el ejercicio físico no cambia la masa libre de grasa, pero el tipo e intensidad de ejercicio no fue contabilizado y no se reportaron los resultados de la medición de masa muscular (emplearon BIA o DXA y reportaron masa libre de grasa), lo que impide determinar si el tipo y/o intensidad del ejercicio tendrá algún efecto aditivo o protector sobre la masa muscular. Por lo anterior, futuros estudios deberán cuantificar esta variable con el fin de evaluar el efecto conjunto de la dieta cetogénica más ejercicio y vislumbrar si existe un posible riesgo de sarcopenia u osteopenia $^{(37-39)}$.

Según nuestra revisión, la dieta cetogénica induce una pérdida de peso, con una disminución modesta en el IMC y la masa grasa. Es por esto por lo que frente a la falta de evidencia contundente sobre el impacto de la dieta cetogénica en la composición corporal, en particular sobre la masa muscular, los investigadores consideramos que es innecesario exponer a los pacientes con exceso de peso a este régimen alimentario. Además, es necesario tener en cuenta el hecho de que la dieta cetogénica impone un mayor gasto de recursos económicos, genera cambios drásticos en el estilo de vida, un alto riesgo de sufrir deficiencias de micro y/o macronutrientes y ocasiona adaptaciones metabólicas, las cuales están contraindicadas en diversas condiciones como la diabetes mellitus tipo 1, enfermedades hepáticas, insuficiencia renal, depresión, entre otras. La evidencia sobre el tratamiento médico nutricional, incluyendo una alimentación hipocalórica y estilos de vida saludables, pueden generar a largo plazo los mismos resultados en la disminución del peso y cambios en la composición corporal $^{(1,2,40)}$.

\section{LIMITACIONES}

Este estudio presenta varias fortalezas: es el primer metaanálisis que evalúa los cambios en varios componentes de la composición corporal; identifica el efecto individual y combinado de la dieta cetogénica con ejercicio; contempla análisis estadísticos según la duración del seguimiento; evalúa la diferencia de restricción calórica entre la dieta cetogénica y la dieta hipocalórica; y no involucra estudios relacionados a dietas bajas en carbohidratos, las cuales, en ocasiones, no cumplen con los criterios de una dieta cetogénica baja en calorías.

Una limitación proveniente desde los estudios primarios que condiciona este y los anteriores metaanálisis es que las investigaciones se han realizado bajo condiciones controladas y de seguimiento, por lo que estudios pragmáticos o en ambientes no controlados podrían arrojar resultados diferentes; por esto, futuras investigaciones deberían evaluar el efecto bajo condiciones de la práctica rutinaria sin relegar la necesidad de evaluar la adherencia a la intervención.

Una segunda limitación en los estudios primarios se relaciona con que no se empleó un criterio homogéneo de dieta cetogénica, lo cual explicaría la diferencia en la precisión de los intervalos de confianza respecto a los metaanálisis previos. Finalmente, el sesgo de reporte y la falta de respuesta por algunos autores influencian los resultados de cualquier metaanálisis; esto no fue la excepción en la presenta investigación. Por lo tanto, sugerimos que los futuros metaanálisis y revisiones sistemáticas no utilicen como criterio de selección la falta de información y mejor procuren resaltar la falta 
de información en el campo de estudio, con el fin de optimizar la buena práctica en el reporte de la investigación científica.

\section{CONCLUSIONES}

En adultos con sobrepeso u obesidad, la dieta cetogénica baja en calorías, en comparación con la dieta hipocalórica, presenta una mayor reducción de peso corporal y una leve reducción del IMC y la masa grasa; sin embargo, estos hallazgos pueden estar influenciados por sesgos de reporte y de publicación. Futuros estudios sobre la dieta cetogénica baja en calorías deben enfocarse en el efecto aditivo de la actividad física, los efectos sobre la masa muscular en la medición de la composición corporal; además, deben realizarse en ambientes más cercanos a la práctica habitual y evitar el sesgo de reporte. Finalmente, por los resultados expuestos y el conocimiento acumulado en el campo de investigación, se considera innecesario exponer a los pacientes con sobrepeso a una dieta cetogénica debido a que los cambios en el peso y la composición corporal se pueden obtener con planes más ajustados a una alimentación saludable normal.

\section{PUNTOS CLAVE}

- Metaanálisis previos se centran en analizar los cambios de peso corporal de la dieta cetogénica baja en calorías.

- La dieta cetogénica permite una mayor reducción del peso corporal y del índice de masa corporal (IMC) respecto a la dieta hipocalórica estándar.

- La dieta cetogénica presentó una reducción leve de masa grasa respecto a la dieta hipocalórica. Sin embargo, no hay información para evaluar el efecto de la dieta cetogénica sobre la masa muscular.

- El riesgo de sesgo, el sesgo de reporte y el sesgo de publicación pueden estar sobreestimando los efectos de la dieta cetogénica respecto a la dieta hipocalórica.

- Ante la falta de conocimiento del efecto de la dieta cetogénica sobre la composición corporal y la evidencia sobre la eficacia del tratamiento nutricional convencional, no se recomienda el uso de la dieta cetogénica en la obesidad.

\section{Declaración de autoría}

DG y CA contribuyeron con la concepción y diseño de la investigación; DG realizó la búsqueda de información en las bases de datos. Todos los autores participaron en la recolección, evaluación y extracción de los datos. DG realizó el análisis estadístico de la información. Todos los autores contribuyeron en la interpretación de los datos y la elaboración del manuscrito. Todos los autores revisaron el manuscrito y acuerdan ser plenamente responsables de garantizar la integridad y precisión del trabajo. Todos los autores leyeron y aprobaron el manuscrito final.

\section{Conflicto de intereses}

Los autores declaran no tener conflictos de interés.

\section{Financiación}

El presente estudio no tuvo financiación.

\section{Referencias bibliográficas}

1. Castellana M, Conte E, Cignarelli A, et al. Efficacy and safety of very low calorie ketogenic diet (VLCKD) in patients with overweight and obesity: A systematic review and metaanalysis. Rev Endocr Metab Disord. 2020;21(1):5-16. doi: 10.1007/s11154-019-09514-y

2. Bueno NB, De Melo ISV, De Oliveira SL, et al. Very-lowcarbohydrate ketogenic diet $\mathrm{v}$. low-fat diet for long-term weight loss: A meta-analysis of Randomised controlled trials. Br J Nutr. 2013;110(7):1178-87. doi: 10.1017/ S0007114513000548

3. Fasulo L, Semprino M, Caraballo R. El equipo multidisciplinario en la aplicación clínica de la dieta cetogénica [Multidisciplinary teamwork in the clinical application of the ketogenic diet]. Medicina (B Aires). 2019;79(3):225-231

4. Cederholm T, Barazzoni R, Austin P, et al. ESPEN guidelines on definitions and terminology of clinical nutrition. Clin Nutr. 2017;36(1):49-64. doi: 10.1016/j.clnu.2016.09.004

5. Pamuk B, Yilmaz H, Kebapçilar L, et al. The effect of orlistat and weight loss diet on plasma ghrelin and obestatin. J Res Med Sci. 2018;23(1):95. doi: 10.4103/jrms.JRMS_928_17

6. Nguyen NT, Varela JE. Bariatric surgery for obesity and metabolic disorders: State of the art. Nat Rev Gastroenterol Hepatol. 2017;14(3):160-9. doi: 10.1038/nrgastro.2016.170

7. Painter SL, Ahmed R, Kushner RF, et al. Expert coaching in weight loss: Retrospective analysis. J Med Internet Res. 2018;20(3):e92. doi: 10.2196/jmir.9738

8. Ardavani A, Aziz H, Smith K, et al. The Effects of Very Low Energy Diets and Low Energy Diets with Exercise Training on Skeletal Muscle Mass: A Narrative Review. Adv Ther. 2020;38(1):149-63. doi: 10.1007/s12325-020-01562-0

9. Rondanelli M, Faliva MA, Gasparri C, et al. Current opinion on dietary advice in order to preserve fat-free mass during a low-calorie diet. Nutrition. 2020;72:110667. doi: 10.1016/j. nut.2019.110667 
10. Beaudart C, Zaaria M, Pasleau F, et al. Health Outcomes of Sarcopenia: A Systematic Review and Meta-Analysis. PLoS One. 2017;12(1):e0169548. doi: 10.1371/journal. pone. 0169548

11. Ge L, Sadeghirad B, Ball GDC, et al. Comparison of dietary macronutrient patterns of 14 popular named dietary programmes for weight and cardiovascular risk factor reduction in adults: Systematic review and network meta-analysis of randomised trials. BMJ. 2020;369(9):e230. doi: 10.1136/bmj. $\mathrm{m} 696$

12. Merra G, Gratteri S, De Lorenzo A, et al. Effects of very-lowcalorie diet on body composition, metabolic state, and genes expression: A randomized double-blind placebo-controlled trial. Eur Rev Med Pharmacol Sci. 2017;21(2):329-45.

13. Romano L, Marchetti M, Gualtieri P, et al. Effects of a personalized VLCKD on body composition and resting energy expenditure in the reversal of diabetes to prevent complications. Nutrients. 2019;11(7):1526. doi: 10.3390/nu11071526

14. Gomez-Arbelaez D, Crujeiras AB, Castro AI, et al. Acid-base safety during the course of a very low-calorie-ketogenic diet. Endocrine. 2017;58(1):81-90. doi: 10.1007/s12020-0171405-3

15. Feinman $\mathrm{RD}$. The biochemistry of low-carbohydrate and ketogenic diets. Curr Opin Endocrinol Diabetes Obes. 2020;27(5):261-8. doi: 10.1097/MED.0000000000000575

16. Di Rosa C, Lattanzi G, Taylor SF, et al. Very low calorie ketogenic diets in overweight and obesity treatment: Effects on anthropometric parameters, body composition, satiety, lipid profile and microbiota. Obes Res Clin Pract. 2020;14(6):491503. doi: 10.1016/j.orcp.2020.08.009

17. Paoli A, Moro T, Bosco G, et al. Effects of $n-3$ polyunsaturated fatty acids $(\omega-3)$ supplementation on some cardiovascular risk factors with a ketogenic mediterranean diet. Mar Drugs. 2015;13(2):996-09. doi: 10.3390/md13020996

18. Moreno B, Crujeiras AB, Bellido D, et al. Obesity treatment by very low-calorie-ketogenic diet at two years: reduction in visceral fat and on the burden of disease. Endocrine. 2016;54(3):681-90. doi: 10.1007/s12020-016-1050-2

19. Lee HS, Lee J. Effects of Combined Exercise and Low Carbohydrate Ketogenic Diet Interventions on Waist Circumference and Triglycerides in Overweight and Obese Individuals: A Systematic Review and Meta-Analysis. Int J Environ Res Public Health. 2021;18(2):828. doi: 10.3390/ ijerph18020828

20. Naude CE, Schoonees A, Senekal M, et al. Low carbohydrate versus isoenergetic balanced diets for reducing weight and cardiovascular risk: A systematic review and meta-analysis. PLoS One. 2014;9(7):e100652. doi: 10.1371/journal. pone. 0100652

21. Gomez-Arbelaez D, Bellido D, Castro AI, et al. Body composition changes after very-low-calorie ketogenic diet in obesity evaluated by 3 standardized methods. J Clin Endocrinol Metab. 2017;102(2):488-98. doi: 10.1210/jc.2016-2385
22. Perticone M, Maio R, Sciacqua A, et al. Ketogenic dietinduced weight loss is associated with an increase in Vitamin D levels in obese adults. Molecules. 2019;24(13):2499. doi: 10.3390/molecules 24132499

23. Sajoux I, Lorenzo PM, Gomez-Arbelaez D, et al. Effect of a very-low-calorie ketogenic diet on circulating myokine levels compared with the effect of bariatric surgery or a low-calorie diet in patients with obesity. Nutrients. 2019;11(10):2368. doi: $10.3390 /$ nu11102368

24. Saslow L, Daubenmier J, Moskowitz J, et al. Twelve-month outcomes of a randomized trial of a moderate-carbohydrate versus very low-carbohydrate diet in overweight adults with type 2 diabetes mellitus or prediabetes. Nutr Diabetes. 2017; (7):304. doi: 10.1038/s41387-017-0006-9

25. Goday A, Bellido D, Sajoux I, et al. Short-Term safety, tolerability and efficacy of a very low-calorie-ketogenic diet interventional weight loss program versus hypocaloric diet in patients with type 2 diabetes mellitus. Nutr Diabetes. 2016;6(9):e230. doi: $10.1038 /$ nutd.2016.36

26. Rubini A, Bosco G, Lodi A, et al. Effects of Twenty Days of the Ketogenic Diet on Metabolic and Respiratory Parameters in Healthy Subjects. Lung. 2015;193(6):939-45. doi: 10.1007/ s00408-015-9806-7

27. Hussain T, Mathew T, Dashti A, et al. Effect of low-calorie versus low-carbohydrate ketogenic diet in type 2 diabetes. Nutrition. 2012;28:1016-21. doi: 10.1016/j.nut.2012.01.016

28. Westman E, Yancy W, Mavropoulos J, et al. The effect of a lowcarbohydrate, ketogenic diet versus a low-glycemic index diet on glycemic control in type 2 diabetes mellitus. Nutr Metab (Lond). 2008; (5):36. doi: 10.1186/1743-7075-5-36

29. Johnston CS, Tjonn SL, Swan PD, et al. Ketogenic low-carbohydrate diets have no metabolic advantage over nonketogenic low-carbohydrate diets. Am J Clin Nutr. 2006;83(5):1055-61. doi: 10.1093/ajcn/83.5.1055

30. Yancy WS, Olsen MK, Guyton JR, et al. A Low-Carbohydrate, Ketogenic Diet versus a Low-Fat Diet to Treat Obesity and Hyperlipidemia: A Randomized, Controlled Trial. Ann Intern Med. 2004;140(10):769-77. doi: 10.7326/0003-4819-14010-200405180-00006

31. Mansoor N, Vinknes KJ, Veierod MB, et al. Effects of lowcarbohydrate diets $\mathrm{v}$. low-fat diets on body weight and cardiovascular risk factors a meta-analysis of randomised controlled trials. Br J Nutr. 2016;115(3):466-79. doi: 10.1017/ S0007114515004699

32. Sackner-Bernstein J, Kanter D, Kaul S. Dietary intervention for overweight and obese adults: comparison of low- carbohydrate and low-fat diets. a meta-analysis. PLoS One. 2015;10(10):e0139817. doi: 10.1371/journal.pone.0139817

33. Tobias DK, Chen M, Manson JAE, et al. Effect of low-fat diet interventions versus other diet interventions on long-term weight change in adults: A systematic review and meta-analysis. Lancet Diabetes Endocrinol. 2015;3(12):968-79. doi: $10.1016 /$ S2213-8587(15)00367-8 
34. Canuto R, Garcez A, de Souza RV, et al. Nutritional intervention strategies for the management of overweight and obesity in primary health care: A systematic review with meta-analysis. Obes Rev. 2021;22(3):e13143. doi: 10.1111/obr.13143

35. Fechner E, Smeets ETHC, Schrauwen P, et al. The effects of different degrees of carbohydrate restriction and carbohydrate replacement on cardiometabolic risk markers in humans - a systematic review and meta-analysis. Nutrients. 2020;12(4):991. doi: 10.3390/nu12040991

36. Choi YJ, Jeon SM, Shin S. Impact of a ketogenic diet on metabolic parameters in patients with obesity or overweight and with or without type 2 diabetes: A meta-analysis of randomized controlled trials. Nutrients. 2020;12(7):1-19. doi: $10.3390 /$ nu12072005
37. Poggiogalle E, Parrinello E, Barazzoni R, et al. Therapeutic strategies for sarcopenic obesity: a systematic review. Curr Opin Clin Nutr Metab Care. 2021;24(1):33-41. doi: 10.1097/ MCO.0000000000000714

38. Paoli A, Cancellara P, Pompei P, et al. Ketogenic diet and skeletal muscle hypertrophy: A Frenemy relationship? J Hum Kinet. 2019;68(1):233-47. doi: 10.2478/hukin-2019-0071

39. Nakao R, Abe T, Yamamoto $S$, et al. Ketogenic diet induces skeletal muscle atrophy via reducing muscle protein synthesis and possibly activating proteolysis in mice. Sci Rep. 2019;9(1):19614-52. doi: 10.1038/s41598-019-56166-8

40. Churuangsuk C, Griffiths D, Lean MEJ, et al. Impacts of carbohydrate-restricted diets on micronutrient intakes and status: A systematic review. Obes Rev. 2019;20(8):1132-47. doi: $10.1111 /$ obr.12857 